\title{
Nanocavities cause a stir
}

\section{C6 \\ the formation \\ of nanocavities \\ is central to \\ concentrating \\ the immiscible reactants}

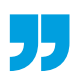

Reactions between gases and liquids are important in industry but are typically slow and difficult to monitor in situ. Elevated temperatures and pressures are often required to increase the efficiency of reactions between immiscible gases and liquids. Now, reporting in Angewandte Chemie International Edition, Xing Yi Ling and colleagues introduce a platform composed of an array of solid nanoparticles coated in a metal-organic framework (MOF) that substantially increases gas-liquid reaction efficiency owing to the formation of interfacial nanocavities. The platform can also be adapted for the in situ monitoring of reactions.

Increasing the frequency of collisions between gas and liquid molecules is key to achieving highly efficient gas-liquid reactions. Ling and co-workers approach this problem by designing a system that concentrates gas and liquid molecules in nanoscale cavities formed at the interface between a MOF sorbent and a solid surface (solid@MOF). Ling compares the
solid@MOF ensemble to a vacuum cleaner: "the MOF serves as the vacuum generator to continuously 'suck' in gas and liquid molecules, and the nanoscale cavities function as the collection bag to trap these molecules." Each nanocavity is a pocket of pseudo high pressure that promotes the gas-liquid reaction.

The team fabricate their platform by growing a thin film of zeolitic imidazolate framework-8 (ZIF) over a 2D array of Ag nanocubes. The latter were chosen for their plasmonic activity, which enables the use of surfaceenhanced Raman spectroscopy (SERS) to probe a reaction at the Ag-ZIF interface. "On-site reaction monitoring is crucial, especially when reaction species are unstable towards post-reaction treatments," remarks Ling. The Ag@ZIF platform was assessed for its activity towards $\mathrm{CO}_{2}$ and aniline, two reagents that produce phenylcarbamic acid in a reaction that is typically initiated by a catalyst and/or raised temperature or pressure. Ling and co-workers

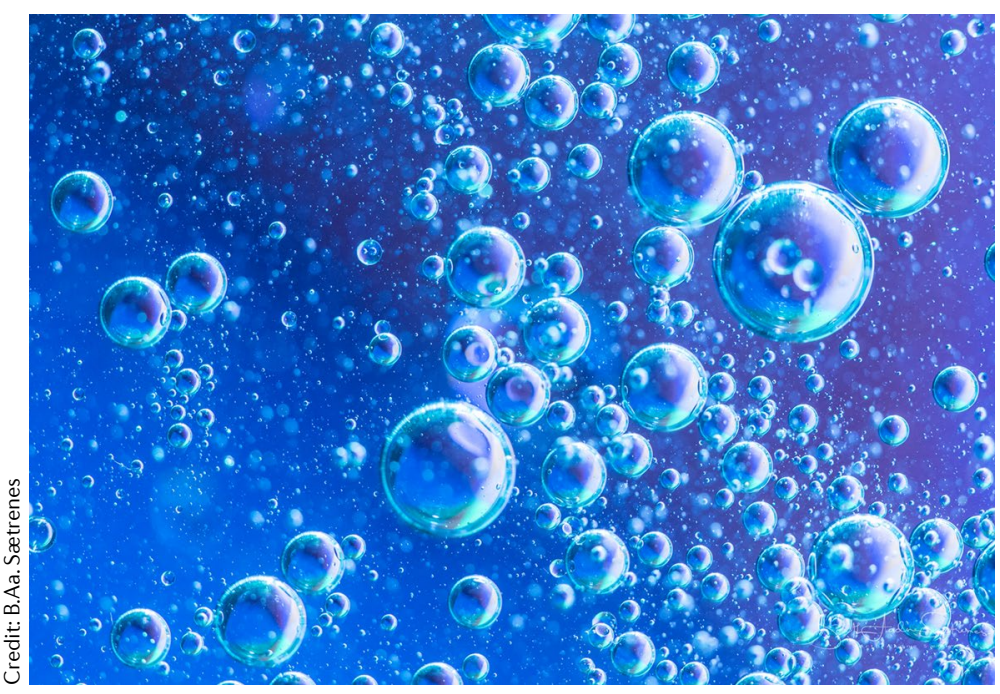

bubbled $\mathrm{CO}_{2}$ gas through a reaction cell containing the Ag@ZIF platform immersed in an aniline solution at room temperature. Real-time monitoring using SERS indicated the formation of phenylcarbamic acid in a reaction that would otherwise not proceed at ambient conditions.

In addition to ZIF-coated Ag nanoarrays, Ling's team fabricated Ag@ZIF core-shell nanoparticles, which have a larger interfacial area and higher efficiency in the reaction between $\mathrm{CO}_{2}$ and aniline. Moreover, after normalizing for the mass of ZIF, the reaction efficiency of the Ag@ZIF core-shell nanoparticles is shown to be at least 28 times higher than that of ZIF-only crystallites. This finding not only highlights that interfacial area is more important than the mass of MOF, but crucially also demonstrates that the formation of nanocavities is central to concentrating the immiscible reactants and subsequently driving their reaction.

Given the straightforwardness of the solid@MOF approach, the team is currently investigating alternative solid substrate materials with the aim of increasing the efficiency of gas-liquid reactions under ambient conditions. Plasmonic solid@MOF ensembles may also provide hitherto unobtainable insight into multiphasic reactions. "With a vast library of available MOF and functional solid substrates, we expect many potential applications for solid@MOF systems,” says Ling.

Claire Ashworth

ORIGINAL ARTICLE Sim, H. Y. F. et al. Concentrating immiscible molecules at solid@ MOF interfacial nanocavities to drive an inert gas-liquid reaction at ambient conditions. Angew. Chem. Int. Ed. https://doi.org/10.1002/ anie.201809813 (2018) 